\title{
Assessment the Effect of Prophylaxis Therapy on Splenic and Portal Vein Thrombosis after Open Splenectomy: A Controlled Randomized Clinical Trial
}

\author{
Amin Bahreini ${ }^{1}$, Majid Asnaashari ${ }^{2}$, Amir Ahmad Salmasi ${ }^{3} \&$ Mohamad Momen Gharibvand $^{2}$ \\ ${ }^{1}$ Department of General Surgery, Ahvaz Jundishapur University of Medical Science, Ahvaz, Iran \\ ${ }^{2}$ Department of Radiology, Ahvaz Jundishapur University of Medical Science, Ahvaz, Iran \\ Correspondence: Dr Majid Asna Ashari, Azedegan Boulevard, Imam Khomeini Hospital, Ahvaz Jundishapur \\ University of Medical Sciences, Ahvaz, Iran. Tel: 98-912-648-3790. E-mail: drasnaashari60@gmail.com
}

Received: July 6, 2016 Accepted: September 2, 2016 Online Published: October 18, 2016

doi:10.5539/gjhs.v9n5p189 URL: http://dx.doi.org/10.5539/gjhs.v9n5p189

\begin{abstract}
Splenic and portal vein thrombosis (SPVT) is considered as a serious complication of splenectomy with potential life-threatening. Chemoprophylaxis may help to curb the incidence of SPVT after splenectomy. This clinical trial study was conducted to determine the incidence rate of SPVT after splenectomy and investigate the effect of chemoprophylaxis to reduce its incidence. Sixty six patients undergoing open splenectomy were included in this single-blind clinical randomized controlled trial (RCT). Patients were randomly assigned in two groups of intervention and control using block randomization to either d receive $40 \mathrm{mg}$ of enoxaparin subcutaneously once a day for 5 days and then $100 \mathrm{mg}$ aspirin for one month or no postoperative drug. After one month, all patients over a week underwent Doppler ultrasonography of the splenic, portal and superior mesenteric veins for thrombosis. The mean age of patients was similar between intervention and control groups ( $28.3 \pm 14.5$ and $25.6 \pm 14.9$, respectively) $(\mathrm{P}$ value $=0.9$ ).Furthermore, two groups were matched regarding distribution of gender. None of patients in intervention group developed portal vein thrombosis, while of 23 patients in control group, $2(8.69 \%)$ subjects were diagnosed with portal vein thrombosis. The two groups had no statistically significant difference in the rate of portal vein thrombosis $(\mathrm{P}=0.18)$. Based on the results of our study, prophylaxis therapy had no effects in preventing portal vein thrombosis developed in patients undergoing open splenectomy for any reason.
\end{abstract}

Keywords: open splenectomy, portal vein thrombosis, prophylaxis

\section{Introduction}

The first splenectomy was performed in Naples by Andirano Zacarelli in 1549 on a young woman for splenomegaly (Fujita, Lyass, Otsuka, \& Giordano, 2003; Ikeda et al., 2005; Stamou et al., 2006; Tran et al., 2010; Winslow, Brunt, Drebin, Soper, \& Klingensmith, 2002). After that, splenectomy is considered as a therapeutic approach for various clinical conditions including ruptured spleen, WBC and platelet and RBC disorders, hemoglobinopathies, bone marrow disorders, cysts, tumors, infections, abscesses, storage diseases and other disorders. In general, the most frequent indication for splenectomy is splenic trauma, as external trauma or iatrogenic injury during surgery (Smink, 2015). Complications after splenectomy are left lung lobe atelectasis, pneumonia, pulmonary edema, intraoperative and postoperative hemorrhage, sub-diaphragmatic abscess, pancreatitis, pancreatic fistula, pancreatic pseudocyst, deep vein thrombosis and ultimately splenic, superior mesenteric and portal veins thrombosis (Cadili \& de Gara, 2008; James et al., 2009; Targarona, 2008). Splenic and portal vein thrombosis (SPVT) is a potentially life-threatening complication of splenectomy, which is characterized by portal, mesenteric and splenic venous clot propagates, and splenectomy is one of the most common causes. Retrospective and prospective studies have suggested between $8 \%$ and $24 \%$ incidence for asymptomatic SPVT and between 0 and 19\% for symptomatic SPVT (Wang, Kopac, Brisebois, Sample, \& Shapiro, 2011). Clinical signs of SPVT include portal vein hypertension as esophageal varices, upper gastrointestinal bleeding (UGIB) and hypersplenism and intestinal ischemia (Janssen et al., 2001). The risk factors for SPVT include lymphoma, lymphoproliferative disorder, hemolytic anemia associated with splenomegaly (more than 650 grams), splenic vein diameter greater than $8 \mathrm{~mm}$ and hyper-coagulopathy (Qi, Bai, Guo, \& Fan, 2014; Schettino, Fagundes, Roquete, Ferreira, \& Penna, 2006). In addition, high possibility of SPVT can be seen in laparoscopic 
splenectomy. Several studies have shown that CT scan and color Doppler ultrasound in routine examinations could indicate early thrombosis in SPVT (Wang et al., 2011).

Chemoprophylaxis may help to reduce the incidence of SPVT after splenectomy and thereby reducing complications (Qi et al., 2014). The routine anticoagulants after splenectomy have not been yet standardized. European clinical practice guidelines propose the use of SC heparin as prophylaxis in all patients, unlike the new surgical guidelines (Wang et al., 2011). However, low-dose fondaparinux has been proposed for patients with high-risk LMWH (Low molecular weight heparins) (Wang et al., 2011; Qi et al., 2014). There are underpowered studies to determine the role of prophylaxis in preventing SPVT after splenectomy (Wang et al., 2011). For this reason, this clinical trial study was designed to determine the incidence rate of SPVT after splenectomy and demonstrate the effect of chemoprophylaxis in reducing the incidence.

\section{Materials and Methods}

This single-blind clinical randomized controlled trial (RCT) was conducted to evaluate the effect of prophylactic anticoagulation and antiplatelet in preventing the occurrence of venous thrombosis in portal venous system after open splenectomy. This study was approved by Ethical Committee of Ahvaz Jundishapur University of Medical Sciences, Iran, (ID number: IR.ajums.rec.1394.73866). In addition, this study was registered at the website of www.irct.ir with registration number of IRCT2016042527600N1. The patients underwent open splenectomy, for any reason, in Ahvaz Golestan Hospital were selected in 2015, and the aim of the design and implementation of therapeutic approach were explained to them. Inclusion criteria in this study were patients underwent open splenectomy for any reason and exclusion criteria were uncooperative of patient during ultrasound, history of thromboembolism and death. Then, written consent was obtained from the patient and relevant information was recorded in accordance with the form provided. The patients were divided into two intervention and control groups by block randomization. Basic color Doppler ultrasound was performed by a radiologist for portal vein thrombosis for all patients. Routine preoperative and postoperative measures were carried out on both groups and open splenectomy surgery was performed in the usual way.

Totally, at our center, only 66 patients eligible for inclusion in the study underwent open splenectomy during 2015. Postoperatively, those assigned to the intervention group $(n=33)$ received $40 \mathrm{mg}$ of enoxaparin subcutaneously once a day for 5 days and then $100 \mathrm{mg}$ aspirin for one month; those in the control group $(\mathrm{n}=33)$ received no postoperative drug. After a month, all patients over a week underwent color Doppler ultrasound for the splenic, portal and superior mesenteric veins for detecting thrombosis. The results were recorded and finally the two groups were followed-up for the incidence of portal vein thrombosis. In order to minimize observer bias, the person performing the ultrasound of patients was kept without awareness, indicating single-blinded clinical trial.

To analyze the data, the studied variables were described using descriptive statistical methods including frequency distribution, figures, and central tendency as well as dispersion measures. The Kolmogorov - Smirnov test was used for normality of quantitative variables. Chi-square and independent $t$ or Mann-Whitney statistical tests were used to investigate the relationship between the qualitative and quantitative variables in the groups. Data was analyzed using the SPSS 20.0 software P-values $<0.05$ was deemed as statistically significant.

\section{Results}

In total, 66 patients were included for the analysis. The comparison between two groups regarding background data and the main outcome was presented in Table 1 . The study participants were 47 males (71.2\%) and 19 females $(28.8 \%)$ with mean age of $26.9 \pm 14.7$ years ranged from 6 to 68 years. The intervention group included 24 males $(72.7 \%)$ and 9 females $(27.3 \%)$ with mean age of $28.3 \pm 14.5$ years, and the control group consisted of 23 males $(69.7 \%)$ and 10 female (30.3\%) with mean age of $25.6 \pm 14.9$ years. No statistically significant differences were observed between the two groups for age $(\mathrm{P}=0.9)$ and gender $(\mathrm{P}=0.7)$.

Underlying diseases leading to splenectomy in the intervention group included: 14 cases with trauma (42.4\%), 7 cases with thalassemia major (21.2\%) and 12 cases with other diseases (36.4\%) such as sickle cell anemia, ITP (idiopathic thrombocytopenic), lymphoma, gastric cancer, splenic abscess, splenic and pancreatic cysts; and 14 cases with trauma (42.4\%), 11 cases with thalassemia major (33.3\%) and 8 cases with other diseases $(24.2 \%)$ in control group, indicating no statistically significant differences between the two groups $(\mathrm{P}=0.4)$. The hospitalized duration was $3.7 \pm 1.3$ days in case group and $3.2 \pm 0.7$ days in the control group that this difference was statistically significant $(\mathrm{P}=0.01)$. The difference and the mean hospitalized duration in the case group were higher than control group. The majority of patients ( 37 subjects, $56.1 \%$ ) had been hospitalized about three days.

Forty seven (71.2\%) patients showed perfect cooperation, 5 (7.6\%) had a poor cooperation and $14(21.2 \%)$ had no required cooperation until the end of the study for various reasons and so their information was eliminated from the 
final analysis. In the intervention group, of 33 study participants, 24 (72.7\%) patients had complete cooperation, 5 patients (15.2\%) had poor cooperation (avoiding the use of drugs as a whole), one patients was excluded from the study due to immigration to another city, one patients was excluded due to death during the study, and 2 patients were excluded from the analysis due to other reasons. Ultimately the collected data of these 29 patients (87.9\%) was included in the final calculations. In the control group, 23 (69.7\%) of 33 subjects showed full cooperation and so their data was entered into the final analysis, and 10 (30.3\%) were excluded for various reasons; 6 failure to cooperation, 2 immigrations and 2 deaths during the study due to CVA and trauma. Finally, 52 subjects including 29 in the case group and 23 in the control group were evaluated for splenic, superior mesenteric and portal veins thrombosis through color Doppler ultrasound; 2 patients $(8.69 \%)$ of the 23 patients in control group who had thalassemia major underwent open splenectomy were diagnosed with portal vein thrombosis. None of the patients in the intervention group had venous thrombosis in three mentioned veins. The two groups had no statistically significant difference in the incidence rate of portal vein thrombosis $(\mathrm{P}=0.18)$.

Table 1. Comparison of clinical and consequences data of patients participating in the study

\begin{tabular}{|c|c|c|c|c|}
\hline \multicolumn{2}{|l|}{ Variable } & \multirow{2}{*}{$\begin{array}{l}\text { Intervention group } \\
(\mathrm{n}=33) \\
24(72.2 \%)\end{array}$} & \multirow{2}{*}{$\begin{array}{l}\text { Control group } \\
(\mathrm{n}=33)\end{array}$} & P value \\
\hline \multirow{2}{*}{ Gender } & Male & & & \multirow{2}{*}{0.7} \\
\hline & Female & $19(28.8 \%)$ & $10(30.3 \%)$ & \\
\hline \multicolumn{2}{|c|}{ Mean Age (year) } & $28.3 \pm 14.5$ & $25.6 \pm 14.9$ & 0.9 \\
\hline \multirow{3}{*}{$\begin{array}{l}\text { Underlying } \\
\text { diseases }\end{array}$} & Trauma & $14(42.2 \%)$ & $14(42.2 \%)$ & \multirow{3}{*}{0.4} \\
\hline & Major Thalassemia & $7(21.2 \%)$ & $11(33.3 \%)$ & \\
\hline & Other & $12(36.4 \%)$ & $8(24.4 \%)$ & \\
\hline \multicolumn{2}{|c|}{ Hospitalized Duration (day) } & $3.73 \pm 1.35$ & $3.27 \pm 0.76$ & 0.01 \\
\hline \multirow{3}{*}{ Spleen size } & Normal & $19(57.6 \%)$ & $15(45.5 \%)$ & \multirow{3}{*}{0.9} \\
\hline & Enlarged & $6(18.2 \%)$ & $8(24.2 \%)$ & \\
\hline & Massive & $8(24.2 \%)$ & $10(30.3 \%)$ & \\
\hline \multirow{3}{*}{ Cooperation } & Perfect & $24(72.2 \%)$ & $23(69.7 \%)$ & \multirow{3}{*}{-} \\
\hline & Poor & $5(15.2 \%)$ & 0 & \\
\hline & No cooperation & $4(12.1 \%)$ & $10(30.3 \%)$ & \\
\hline
\end{tabular}

The size of spleen according to different underlying diseases is shown in Table 2. Normal spleen size was up to $15 \mathrm{~cm}$, splenomegaly was considered as bigger than normal size (enlarged spleens), and spleen size greater than $22 \mathrm{~cm}$ was considered as massive spleen. Nineteen $(57.6 \%)$ normal spleens, $6(18.2 \%)$ enlarged spleens and 8 (24.2\%) massive spleens were observed among patients in the intervention group. Fifteen (45.5\%), 8 (24.2\%) and $10(30.3 \%)$ of patients had normal, large and massive spleens in the control group, respectively. In this regard, the two groups had no statistically significant difference $(\mathrm{P}=0.9)$. All patients with trauma in the intervention group $(100 \%)$ and $12(85.7 \%)$ patients in the control group had normal spleens. Only $2(14.3 \%)$ patients with trauma in control group had enlarged spleens, whereas $5(71.4 \%)$ patients with thalassemia major in the intervention group and $8(72.7 \%)$ in the control group had massive spleen. The differences in the two groups were statistically significant $(\mathrm{P}=0.001)$. The spleen size would significantly increase from trauma toward the other diseases and ultimately thalassemia major. However, majority of patients (34 cases $-51.5 \%$ ) had normal spleen size. 
Table 2. The size of spleen based on the type of underlying disease

\begin{tabular}{llll}
\hline Size of spleen/underlying disease & Normal & Enlarged & Massive \\
\hline Trauma & $26(92.9 \%)$ & $2(7.1 \%)$ & - \\
Major Thalassemia & $1(5.6 \%)$ & $4(22.2 \%)$ & $1(72.2 \%)$ \\
Other & $7(35 \%)$ & $8(40 \%)$ & $5(25 \%)$ \\
\hline
\end{tabular}

Despite two patients with portal vein thrombosis who were male, no significant association was seen among patients in the control group for the underlying disease and risk of portal vein thrombosis $(\mathrm{P}=0.12)$ as well as for gender $(\mathrm{P}=0.34)$. One of the patients with portal vein thrombosis was 6-year-old boy suffering from thalassemia major with massive spleen size, who had no symptoms at the time of observed thrombosis. Another patient with portal vein thrombosis was 29 -year-old man suffering from thalassemia major with massive spleen size that a month after the surgery and before the ultrasound was admitted to the hospital because of generalized abdominal pain; portal vein thrombosis was raised for him in the examination and undergone repeated laparotomy and resection of the small intestine due to exacerbation of pain (Table 3). After surgery, the patient was discharged from the hospital with good general conditions.

Table 3. Data of patients with portal vein thrombosis in control group

\begin{tabular}{|c|c|c|c|c|c|c|}
\hline Patient No. & $\begin{array}{l}\text { Portal Vein } \\
\text { Thrombosis }\end{array}$ & Gender & Age & Underlying Disease & Size of Spleen & $\begin{array}{l}\text { hospitalized } \\
\text { duration (day) }\end{array}$ \\
\hline Patient 1 & + & $\mathrm{M}$ & 6 & Major Thalassemia & Massive & 3 \\
\hline Patient 2 & + & M & 29 & Major Thalassemia & Massive & 3 \\
\hline
\end{tabular}

\section{Discussion}

The aim of our study was to evaluate the effect of prophylaxis therapy with enoxaparin and aspirin on the incidence of venous thrombosis in portal and splenic veins after open splenectomy. Based on the results of our study, prophylaxis therapy had no effect in preventing portal vein thrombosis developed in patients undergoing open splenectomy for any reason.

Conditions reducing blood flow to the port are associated with extrahepatic portal vein thrombosis (Janssen et al., 2001). Portal vein thrombosis is a life-threatening complication known for splenectomy, but is still as a rare effect of this surgery (Horowitz, Smith, Weber, Rodriguez-Bigas, \& Petrelli, 1996; Mohren et al., 2004). In a study of Rattner et al. (Rattner, Ellman, \& Warshaw, 1993) on 1000 patients, the incidence of symptomatic PSVT was obtained only $0.7 \%$ and reported $2 \%$ by Vant Riet et al. (van't Riet et al., 2000). In these studies, more than 40 percent of splenectomy had been performed followed by trauma, whereas in a study of Romano et al. (Romano, Caprotti, Scaini, et al., 2006) who surveyed splenectomy after hematological diseases, the incidence of venous thrombosis was 7.6\%. Watters et al. (Watters et al., 2010) reported that the incidence of thrombosis after splenectomy was $5 \%$ in patients with trauma. In a study of Hassan et al. (Hassn, Al - Fallouji, Ouf, \& Saad, 2000), the ratio of portal vein thrombosis after open splenectomy has been reported between $4.7 \%$ and $6.6 \%$. In our study, more than $42 \%$ of patients had been undergone splenectomy after the damages caused by the trauma and the incidence rate of venous thrombosis was $3.8 \%$ in all patients in both case and control groups. In the study of Lai et al. (Lai et al., 2012) between 2004 and 2010, 301 subjects all were selected among patients with portal vein hypertension; In general, patients in this study received prophylaxis therapy as weak, regular and strong, which the incidence rate of thrombosis was $30.22 \%$. In accordance with the above results, it appears that the underlying diseases causing splenectomy have an important role in postoperative portal vein thrombosis. According to the findings of Wang et al. (Wang et al., 2011) and Krauth et al. (Krauth, Lechner, Neugebauer, \& Pabinger, 2008), some diseases such as myeloproliferative, lymphoproliferative disorders and genetic hemolytic anemia can significantly increase the incidence of venous thrombosis. Romano et al. (Romano, Caprotti, Conti, et al., 2006) reported that myeloproliferative increases the risk of portal vein thrombosis after splenectomy. Boyle et al. (Boyle, White, Brunson, \& Wun, 2013) found that underlying disorders causing splenectomy are critical factors in developing the venous thrombosis and demonstrated that patients underwent splenectomy because of ITP have shown high risk of venous thrombosis than the control group.

On the other hand, in the study of Dendle et al. (Dendle, Spelman, Sundararajan, Chunilal, \& Woolley, 2015) in 
2015 conducted on 2472 patients in Australia, despite the high incidence of venous thrombosis in patients with hematologic diseases causing splenectomy, no significant differences were observed between these patients and patients with the risk of venous thrombosis caused by trauma; actually these findings are opposite to the results of our study and are consistent with Boyle et al. In our study, there was no association between underlying disease causing splenectomy and the risk of venous thrombosis, but both of patients with portal vein thrombosis had been undergone splenectomy followed by thalassemia major. So it seems that further investigations are needed in this field.

Previous studies showed that the incidence of portal vein thrombosis in open splenectomy varies between $1.6 \%$ and $11 \%$ (Ruiz-Tovar \& Priego, 2016). It should be noted that ultrasonographic evaluation of patients in our study were performed about 1.3 months after surgery; and given that in some studies, thrombosis has been seen in 2 months (7.6\%) (Romano, Caprotti, Conti, et al., 2006) and at least 4 months (22.7\%) (Pietrabissa et al., 2004) and 1 year $(0.7 \%$ and $0.2 \%)$ (Rattner et al., 1993; van't Riet et al., 2000) after the operation, perhaps the increased duration of follow-up caused rising incidence of thrombosis observed in our study (8.6\%). In particular, many patients with venous thrombosis have no symptoms, and the probability of referring to assess is low (Romano, Caprotti, Conti, et al., 2006). Petit et al. (Petit et al., 1994) found in their study that $50 \%$ of patients with portal vein thrombosis remain asymptomatic, and this issue alongside the short-time follow-up in our study can be reduction factors for this complication among patients; in other studies, however, most of SPVTs have been seen between 2 and 22 days after open splenectomy (Winslow et al., 2002). The use of color Doppler ultrasound instead of CT scan with contrast is another factor in our study that could negatively affect the correct diagnosis of venous thrombosis. On the one hand, improved imaging resolution has led to an increase in diagnosis of portal vein thrombosis; on the other hand, the advantage of CT scans has been emphasized in the past in the diagnosis of PSVT after splenectomy (Loring, Panicek, \& Karpeh, 1998; Morasch et al., 2001; Winslow et al., 2002). Ultrasound has its limits in the presence of abdominal fat or bloating to diagnose PSVT, and CT scan gives better results for the diagnosis of thrombosis by eliminating other abdominal organs and concentrate on vessels (Morasch et al., 2001; Winslow et al., 2002).

Moreover, none of patients in intervention group reported adverse side effects associated with enoxaparin and aspirin.

It is recommended that further studies with larger sample size are needed on the incidence rate and the prevention of portal vein thrombosis in patients undergoing splenectomy, especially in patients with thalassemia major.

\section{Conclusion}

According to the results of our study, despite the fact that in the group receiving prophylactic enoxaparin and aspirin no venous thrombosis was observed after open splenectomy, but the drugs had no significant effect in reducing thrombosis; and it does not seem to be advisable as a general treatment for patients undergoing open splenectomy. Also, based on the evidences of other researches and the our findings, it seems necessary to consider the high risk of splenic and portal vein thrombosis in patients with hematologic diseases responsible for splenectomy and especially thalassemia major until the implementation of larger studies.

\section{Acknowledgements}

We would like to give thanks to our study participants for their good cooperation during the study and also Ahvaz Jundishapur University of Medical Sciences for its ethical and financial supports.

\section{Competing Interests Statement}

The authors declare that there is no conflict of interests regarding the publication of this paper.

\section{References}

Boyle, S., White, R. H., Brunson, A., \& Wun, T. (2013). Splenectomy and the incidence of venous thromboembolism and sepsis in patients with immune thrombocytopenia. Blood, 121(23), 4782-4790. http://dx.doi.org/ 10.1182/blood-2012-12-467068.

Wang, H., Kopac, D., Brisebois, R., Sample, C., \& Shapiro, A. M. (2011). Randomized controlled trial to investigate the impact of anticoagulation on the incidence of splenic or portal vein thrombosis after laparoscopic splenectomy. Canadian Journal of Surgery, 54(4), 227. http://dx.doi.org/ 10.1503/cjs.049909.

Cadili, A., \& de Gara, C. (2008). Complications of splenectomy. The American journal of medicine, 121(5), 371-375. http://dx.doi.org/ 10.1016/j.amjmed.2008.02.014.

Dendle, C., Spelman, T., Sundararajan, V., Chunilal, S., \& Woolley, I. (2015). An analysis of the thromboembolic 
outcomes of 2472 splenectomized individuals. Blood, 125(10), 1681-1682. http://dx.doi.org/ 10.1182/blood-2014-12-619122

Fujita, F., Lyass, S., Otsuka, K., \& Giordano, L. (2003). Portal vein thrombosis following splenectomy: identification of risk factors. The American surgeon, 69(11), 951.

Hassn, A., Al-Fallouji, M., Ouf, T., \& Saad, R. (2000). Portal vein thrombosis following splenectomy. British Journal of Surgery, 87(3), 367-368. http://dx.doi.org/ 10.1046/j.1365-2168.2000.01383-16.x.

Horowitz, J., Smith, J. L., Weber, T. K., Rodriguez-Bigas, M. A., \& Petrelli, N. J. (1996). Postoperative complications after splenectomy for hematologic malignancies. Annals of surgery, 223(3), 290.

Ikeda, M., Sekimoto, M., Takiguchi, S., Kubota, M., Ikenaga, M., Yamamoto, H., . . Imamura, H. (2005). High incidence of thrombosis of the portal venous system after laparoscopic splenectomy: a prospective study with contrast-enhanced CT scan. Annals of surgery, 24l(2), 208-216. http://dx.doi.org/ 10.1097/01.sla.0000151794.28392.a6.

James, A. W., Rabl, C., Westphalen, A. C., Fogarty, P. F., Posselt, A. M., \& Campos, G. M. (2009). Portomesenteric venous thrombosis after laparoscopic surgery: A systematic literature review. Archives of Surgery, 144(6), 520-526. http://dx.doi.org/ 10.1001/archsurg.2009.81.

Janssen, H., Wijnhoud, A., Haagsma, E., Van Uum, S., Van Nieuwkerk, C., Adang, R., . . Hansen, B. (2001). Extrahepatic portal vein thrombosis: aetiology and determinants of survival. Gut, 49(5), 720-724. http://dx.doi.org/ 10.1136/gut.49.5.720.

Krauth, M.-T., Lechner, K., Neugebauer, E. A., \& Pabinger, I. (2008). The postoperative splenic/portal vein thrombosis after splenectomy and its prevention-an unresolved issue. Haematologica, 93(8), 1227-1232. http://dx.doi.org/ 10.3324/haematol.12682.

Lai, W., Lu, S.-C., Li, G.-Y., Li, C.-Y., Wu, J.-S., Guo, Q.-L., . . Li, N. (2012). Anticoagulation therapy prevents portal-splenic vein thrombosis after splenectomy with gastroesophageal devascularization. World journal of gastroenterology, 18(26), 3443-3450. http://dx.doi.org/ 10.3748/wjg.v18.i26.3443.

Loring, L. A., Panicek, D. M., \& Karpeh, M. S. (1998). Portal system thrombosis after splenectomy for neoplasm or chronic hematologic disorder: is routine surveillance imaging necessary? Journal of computer assisted tomography, 22(6), 856-860.

Mohren, M., Markmann, I., Dworschak, U., Franke, A., Maas, C., Mewes, S., . . Jentsch - Ullrich, K. (2004). Thromboembolic complications after splenectomy for hematologic diseases. American journal of hematology, 76(2), 143-147. http://dx.doi.org/ 10.1002/ajh.20018.

Morasch, M. D., Ebaugh, J. L., Chiou, A. C., Matsumura, J. S., Pearce, W. H., \& Yao, J. S. (2001). Mesenteric venous thrombosis: a changing clinical entity. Journal of vascular surgery, 34(4), 680-684. http://dx.doi.org/10.1067/mva.2001.116965.

Petit, P., Bret, P. M., Atri, M., Hreno, A., Casola, G., \& Gianfelice, D. (1994). Splenic vein thrombosis after splenectomy: frequency and role of imaging. Radiology, 190(1), 65-68. http://dx.doi.org/ 10.1148/radiology.190.1.8259430.

Pietrabissa, A., Moretto, C., Antonelli, G., Morelli, L., Marciano, E., \& Mosca, F. (2004). Thrombosis in the portal venous system after elective laparoscopic splenectomy. Surgical Endoscopy and Other Interventional Techniques, 18(7), 1140-1143. http://dx.doi.org/ 10.1007/s00464-003-9284-5.

Qi, X., Bai, M., Guo, X., \& Fan, D. (2014). Pharmacologic prophylaxis of portal venous system thrombosis after splenectomy: a meta-analysis. Gastroenterology research and practice, 2014, 292689. http://dx.doi.org/ $10.1155 / 2014 / 292689$.

Rattner, D. W., Ellman, L., \& Warshaw, A. L. (1993). Portal vein thrombosis after elective splenectomy: an underappreciated, potentially lethal syndrome. Archives of Surgery, 128(5), 565-570. http://dx.doi.org/ 10.1001/archsurg.1993.01420170101015.

Romano, F., Caprotti, R., Conti, M., Piacentini, M. G., Uggeri, F., Motta, V., .. . Uggeri, F. (2006). Thrombosis of the splenoportal axis after splenectomy. Langenbeck's Archives of Surgery, 391(5), 483-488. http://dx.doi.org/ 10.1007/s00423-006-0075-z.

Romano, F., Caprotti, R., Scaini, A., Conti, M., Scotti, M., Colombo, G., \& Uggeri, F. (2006). Elective laparoscopic splenectomy and thrombosis of the spleno-portal axis: a prospective study with ecocolordoppler 
ultrasound. Surgical Laparoscopy Endoscopy \& Percutaneous Techniques, 16(1), 4-7. http://dx.doi.org/10.1097/01.sle.0000202187.80407.09

Ruiz-Tovar, J., \& Priego, P. (2016). Portal Vein Thrombosis After Splenic and Pancreatic Surgery. In S. M. Islam (Ed.), Thrombosis and Embolism: from Research to Clinical Practice (Volume 1, pp. 1-11). Cham: Springer International Publishing. http://dx.doi.org/10.1007/13780_2015_7.

Schettino, G., Fagundes, E. D., Roquete, M. L., Ferreira, A. R., \& Penna, F. J. (2006). Portal vein thrombosis in children and adolescents. Jornal de pediatria, 82(3), 171-178. http://dx.doi.org/ 10.2223/JPED.1484.

Smink, D. S. (2015). Schwartz's Principles of Surgery (10th ed.). LWW. http://dx.doi.org/10.1097/SLA.0000000000001107.

Stamou, K. M., Toutouzas, K. G., Kekis, P. B., Nakos, S., Gafou, A., Manouras, A., . . Bramis, J. (2006). Prospective study of the incidence and risk factors of postsplenectomy thrombosis of the portal, mesenteric, and splenic veins. Archives of surgery, 141(7), 663-669. http://dx.doi.org/ 10.1001/archsurg.141.7.663.

Targarona, E. M. (2008). Portal vein thrombosis after laparoscopic splenectomy: the size of the risk. Surgical innovation, 15(4), 266-270. http://dx.doi.org/ 10.1177/1553350608324931.

Tran, T., Demyttenaere, S. V., Polyhronopoulos, G., Séguin, C., Artho, G. P., Kaneva, P., . . \& Feldman, L. S. (2010). Recommended timing for surveillance ultrasonography to diagnose portal splenic vein thrombosis

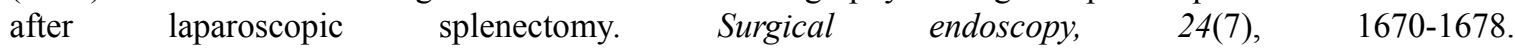
http://dx.doi.org/10.1007/s00464-009-0828-1.

van't Riet, M., Burger, J., Van Muiswinkel, J., Kazemier, G., Schipperus, M., \& Bonjer, H. (2000). Diagnosis and treatment of portal vein thrombosis following splenectomy. British Journal of surgery, 87(9), 1229-1233. http://dx.doi.org/ 10.1046/j.1365-2168.2000.01514.x.

Watters, J. M., Sambasivan, C. N., Zink, K., Kremenevskiy, I., Englehart, M. S., Underwood, S. J., \& Schreiber, M. A. (2010). Splenectomy leads to a persistent hypercoagulable state after trauma. The American Journal of Surgery, 199(5), 646-651. http://dx.doi.org/10.1016/j.amjsurg.2010.01.015.

Winslow, E. R., Brunt, L. M., Drebin, J. A., Soper, N. J., \& Klingensmith, M. E. (2002). Portal vein thrombosis after splenectomy. The American journal of surgery, 184(6), 631-635.

\section{Copyrights}

Copyright for this article is retained by the author(s), with first publication rights granted to the journal.

This is an open-access article distributed under the terms and conditions of the Creative Commons Attribution license (http://creativecommons.org/licenses/by/4.0/). 